\title{
Research on IoT Business Model
}

\author{
Jing Li \\ Xi'an Peihua University, Xi’an, Shaanxi, 710125
}

Keywords: IoT, Business Model, Internet Profit

\begin{abstract}
As another trillion-level market after the communication network, the development of the Internet of Things industry is widely optimistic, but from the current market size development, there is still a big gap between its market size and the prediction of relevant institutions, of which important issues, one is the lack of a business model that adapts to market needs. In this paper, the author uses case study method, interview method and thinking method to analyze foreign mature IoT business model, combine the status of China's industrial development and existing models, and make reasonable and bold predictions and inferences. "The 'dynamic' Internet of Things model" and "cloud" model are expected to promote the further development of the Chinese Internet of Things industry.
\end{abstract}

\section{Introduction}

The term "Internet of Things" was coined in 1999 by Kevin Ashton of MIT (Massachusetts Institute of Technology). As the name suggests, the Internet of Things refers to the connection of the Internet to any item through information-sensing devices such as wireless RF transmitting devices to achieve intelligent identification and management. The Internet of Things has set off a third revolution in the world of information industry following the computer, Internet and mobile communication networks, integrating sensor technology, embedded computer technology, modern network technology and wireless communication technology, distributed information processing technology, etc. These technologies are embedded and equipped into all possible objects of daily life design, and the Internet of Things is connected through these objects to form the Internet of Things, enabling human society to truly enter the era of intelligence and unification. Nowadays, the concept of the Internet of Things has been further improved, and its role has penetrated into all areas of society. As an emerging industry with broad development prospects, the Internet of Things plays a major role in promoting economic development, accelerating the transformation of China's economic development system, and improving China's independent innovation capability. It has become one of the most promising industries. Despite the wide range of applications in the Internet of Things, there are still obstacles in the development of the industry. China's Internet of Things industry is still in its infancy, and a mature, stable, and profitable business model has not yet formed. As a new research field and research object, the Internet of Things is the key to the large-scale and long-term application of the Internet of Things industry. At present, the wave of globalization has swept the world, and market competition has become increasingly fierce. The business model has played an increasingly important role in corporate competition. The famous management scientist Peter Drucker once said: "The competition between enterprises today is not the competition between products, but the competition between business models." From this point of view, the business model is developing in the Internet of Things industry. The importance of the process should not be underestimated. To speed up the development of the Internet of Things, it is imperative to find a mature, stable and profitable business model and improve the core competitiveness of the Internet of Things industry. This is the key to promoting the development of the Internet of Things industry.

\section{Problems in the business model of China's Internet of Things industry}

Although China has achieved certain results in the Internet of Things industry, there is still a 
tortuous road to the real thing in the Internet of Things. There are still many unsuitable business models for the Internet of Things.

The contradiction between scale and cost. At present, the biggest problem in the development of the Internet of Things is the lack of application in the industry and the fragmentation of the business model. The application level of China's Internet of Things is still relatively low. Although there are some basic applications, the market demand is still small-scale and low-level. It cannot be effectively applied in the industrial chain on the Internet and downstream, and ultimately leads to certain To a certain extent, it affects the scale of the Internet of Things. With the continuous promotion of the Internet of Things, its application scope has gradually expanded to various industries and various fields. The application characteristics and user needs of different industries are different. There is no unified standard and norm, which leads to the inability of the Internet of Things to be scaled up. The consequence is that the cost of development, integration, deployment and maintenance of the Internet of Things is relatively high. The small-scale application can not express the great significance of the Internet of Things "IoT", nor can it form a multi-party force to jointly promote the healthy development of the Internet of Things industry, which is undoubtedly not conducive to the sustainable development of the Internet of Things industry. In the process of industrialization of the Internet of Things, small and medium-sized enterprises account for a large proportion, and the leading enterprises with strong strength are relatively lacking, which is not conducive to the demonstration effect of leading enterprises, and is not conducive to the cooperation between enterprises, resulting in the inability of the entire Internet of Things. Effect, higher cost. At the same time, the composition of the IoT industry chain is not perfect, and the upstream enterprises are small in scale and cannot meet the demand of the Internet of Things market in quantity. Some of the mid- and downstream industry chains are relatively mature and have entered the international advanced ranks. This makes the Internet of Things industry unable to form economies of scale, nor can it form an integrated business model, which constrains the development of the Internet of Things industry.

The business model is not clear. At this stage, the development of the Internet of Things industry is in the initial stage of growth, and there are still many problems. As the emerging industry, the Internet of Things has many unstable and incomplete business models. The unclear business model has become an important factor restricting the development of the Internet of Things industry. Due to the rapid advancement of the Internet of Things in China and the lack of a blueprint for a clear overall planning, the development of the current Internet of Things is completely in a spontaneous state, with different regions and different companies fighting each other. The entire industry lacks unified standards and does not form an efficient and clear business model, which makes the development of the Internet of Things industry disorderly and blind. The resources sharing of all parties is insufficient, resulting in serious waste of resources and high research costs, which is very unfavorable to the Internet of Things industry. Great development. At the same time, the structure of the IoT industry chain is very complex, involving terminal manufacturers, application developers, network operators, end users and many other links. The benefits of each link are difficult to distribute. If there is no clear business model, it is difficult to achieve a win-win situation. Leading to unsustainable business models. Therefore, the continuous improvement, innovation and diversification of the business model has become a lasting driving force for the sustainable development of the Internet of Things industry.

The financing of the Internet of Things has become a key issue in its business model. The author believes that if the business model is simply described as a way of making money, then the financing source of the Internet of Things - financing, is the basis for making money. In recent years, the financing of the Internet of Things does not seem to receive widespread attention like other issues in the development of the Internet of Things, because most of the funds for the development of the Internet of Things come from the same subject, which is our government. As an emerging industry, the Internet of Things has caused a lot of controversy in its explosive development. The rapid development and demand are mixed. At the same time, the future development of the Internet of Things is changing, the huge amount of capital demand and the long payback period of revenues 
make the Internet of Things industry financing not so easy. The country has become the owner of the Internet of Things industry. It has formed a single financing object for the Internet of Things industry, and the financing entity is not active enough to fully mobilize the role of the market in the development of the industry.

\section{Internet of Things Industry Business Model Improvement Measures}

Promote cooperation and mutual benefit. In the entire development chain of the IoT business, the advantages of each business entity in terms of capital, management, and technology are not the same. In order to maximize the benefits, it is necessary to promote the full cooperation of all parties in the business. Internet of Things enterprises should encourage all entities in the value chain to play an advantageous role, reduce cost expenditures, achieve maximum value-added, enhance the integration and dynamic balance of business model systems, reduce the cost of participation of all parties involved in the business, and rely on the Internet. Products and services enable revenue growth. Only by combining the strengths of all parties can China's Internet of Things industry flourish, so that the Internet of Things can truly have a long-term, sustainable development momentum, and ultimately achieve a win-win situation.

Government support. The government has a guiding role in the process of enterprise development. The formulation and implementation of government policy guidelines are particularly important for the development of emerging enterprises. As the new darling of today's society, the Internet of Things, in addition to the need for strong market demand and adequate resource allocation, requires the government's support, guidance and the provision of advantageous platforms, so that the Internet of Things will be competitive in the market. . In addition, we must improve the IoT industry policy environment. Through loose market access policies, preferential tax policies and price policies, we will guide various forms of domestic capital to the Internet of Things industry, break down industry barriers, encourage cross-industry investment, guide private capital, and realize the diversity of IoT financing. Then develop a more effective business model.

Technical standardization. The standardization of the Internet of Things industry is the key to overcome the obstacles to the development of the Internet of Things industry and reduce costs. Although the Internet of Things is the third revolutionary wave of the world's information industry, its development is still in its infancy. China should develop independent intellectual property rights technical standards and accelerate pilot applications as soon as possible. With the advantage of its information resources, Wuxi has been given priority and key support as the science and technology demonstration base for the development of the Internet of Things in Jiangsu Province, and has become the leading city for the development of China's Internet of Things. The development of Wuxi Internet of Things reflects the development level of China's Internet of Things to a certain extent. It should play a demonstration role in the technological innovation of the Internet of Things. It is necessary to first overcome technical problems, improve the level of innovation, and establish an industrial incubator base for the Internet of Things. It is specially used for the R\&D and application of the Internet of Things, and finally forms a large-scale industrialization, thereby reducing the cost of the Internet of Things industry, solving the contradiction between cost and scale, improving the profitability of the Internet of Things, developing an effective profit model, and promoting The development of business models.

Establish a broader alliance of cooperation. Representatives of the priority development of the Internet of Things industry such as Wuxi, how to transform into the core advantages of the new round of development, need to create more choices. Wuxi should make full use of all available resource advantages and establish a broader alliance of cooperation among many outstanding universities in Jiangsu. Colleges and universities are the main positions for talent cultivation, providing more possibilities for the innovation of Internet of Things technology, promoting the close connection of industry, education and research in the Internet of Things, improving the level of production, education and research, expanding the scale of production, education and research, and creating a larger development platform. Through cooperation with universities, we can accelerate the development and innovation of the Internet of Things and promote the all-round 
development of the Internet of Things.

\section{Conclusion}

A reasonably effective business model is half the battle. The use of the optimal business model can maximize customer value, integrate the internal and external elements of the company's operations, and form a complete and efficient operating system with unique core competitiveness while meeting customer needs through optimal implementation. To achieve customer value and to make the system achieve the ultimate goal of continuous profitability.

\section{References}

[1] Tao Ye. Exploration and Innovation of the Business Model of the Internet of Things Industry[J].Journal of Nanjing University of Science and Technology, 2010,(4).

[2] Li Zhuoxian. Cloud Aggregation: A Feasible Choice for the Internet of Things Business Model [J]. Communications World Weekly, 2011, (1): 19.

[3] Yuan Changzheng. Analysis of the development of China's Internet of Things industry based on the perspective of industrial economics [J]. Academic Exchange, 2011, (7): 115-118.

[4] Ding Xuejun. Research on Key Issues in the Development of China's Internet of Things Industry [J]. E-commerce, 2011, (5): 8-10.

[5] Wen Liang. Reflections on the problems in the development of China's Internet of Things [J]. Guangdong Science and Technology, 2012, (7): 194-195. 